\title{
Desenvolvendo modelos para o Programa de Promoção da Alimentação Adequada e
}

\section{Saudável: um estudo de avaliabilidade}

\author{
Developing models for the Adequate and Healthy Eating Promotion Program: an evaluability study \\ Desarrollo de modelos para el Programa de Promoción de una Alimentación Adecuada y \\ Saludable: un estudio de evaluabilidad
}

Recebido: 04/08/2021 | Revisado: 13/08/2021 | Aceito: 18/08/2021 | Publicado: 21/08/2021

\author{
Juliana Mara Flores Bicalho \\ ORCID: https://orcid.org/0000-0003-1445-8234 \\ Universidade Federal de São João Del Rei, Brasil \\ E-mail: jmfbicalho@gmail.com \\ Eliete Albano Azevedo Guimarães \\ ORCID: https://orcid.org/0000-0001-9236-8643 \\ Universidade Federal de São João Del Rei, Brasil \\ E-mail: elietealbano@hotmail.com \\ Patrícia Pinheiro de Freitas \\ ORCID: https://orcid.org/0000-0001-9355-3066 \\ Universidade Federal de Minas Gerais, Brasil \\ E-mail: patpfreitas@gmail.com \\ Mariana Souza Lopes \\ ORCID: https://orcid.org/0000-0003-3128-7959 \\ Universidade Federal de Minas Gerais, Brasil \\ E-mail: marianalopes.ufmg@gmail.com \\ Mariana Carvalho de Menezes \\ ORCID: https://orcid.org/0000-0002-3069-7959 \\ Universidade Federal de Ouro Preto, Brasil \\ E-mail: mariana.menezes@ufop.edu.br \\ Aline Cristine Souza Lopes \\ ORCID: https://orcid.org/0000-0001-9782-2606 \\ Universidade Federal de Minas Gerais, Brasil \\ E-mail: alinelopesenf@gmail.com \\ Cláudia di Lorenzo Oliveira \\ ORCID: https://orcid.org/0000-0001-8533-8155 \\ Universidade Federal de São João Del Rei, Brasil \\ E-mail: claudia.dlorenzo@gmail.com
}

\begin{abstract}
Resumo
As doenças crônicas não transmissíveis (DCNT) figuram-se mundialmente como importante problema de saúde pública, sendo a alimentação inadequada um de seus principais determinantes. Dessa forma, a qualificação dos profissionais de saúde para realização de ações de promoção da alimentação adequada e saudável, que sejam práticas, efetivas e viáveis, é essencial. O objetivo deste artigo foi realizar o estudo de avaliabilidade, considerando a descrição da intervenção (implantação do Programa de Promoção da Alimentação Adequada e Saudável - PPAAS na Atenção Primária à Saúde brasileira), a elaboração do modelo lógico e a identificação e validação das questões avaliativas. Adotou-se como referencial o sistema de sete elementos proposto por Thurston e Ramaliu. Foram realizadas análises de documentos, revisão teórica, reuniões para elaboração do modelo teórico lógico do Programa e a identificação das questões avaliativas, que resultou no instrumento de medidas multidimensional para avaliar sua implantação. A validação de conteúdo das questões avaliativas foi realizada por técnica Delphi. O modelo lógico desenvolvido foi essencial para compreender as premissas teóricas básicas do PPAAS, condensando em uma imagem as peculiaridades dos seus componentes e constituindo um produto que pode ser utilizado em pesquisas avaliativas posteriores. O instrumento multidimensional apresentou um índice de validação de conteúdo de $91 \%$. O estudo fornece um modelo lógico e um instrumento multidimensional válidos, imprescindíveis para o planejamento da avaliação propriamente dita da implantação do PPAAS.

Palavras-chave: Programas e políticas de nutrição e alimentação; Educação alimentar e nutricional; Atenção primária à saúde; Avaliação de programas e projetos de saúde.
\end{abstract}




\begin{abstract}
Chronic non-communicable diseases (NCD) are globally an important public health problem, with inadequate nutrition being one of its main determinants. Thus, the qualification of health professionals to carry out actions to promote adequate and healthy eating, which are practical, effective and viable, is essential. The aim of this article was to carry out the evaluability study, considering the description of the intervention (implementation of the Program for the Promotion of Adequate and Healthy Eating - PPAHE in Brazilian Primary Health Care), the elaboration of the logical model and the identification and validation of the evaluative questions. The seven-element system proposed by Thurston and Ramaliu was adopted as a reference. Document analysis, theoretical review, meetings for the elaboration of the logical theoretical model of the Program and the identification of evaluative questions were carried out, which resulted in the instrument of multidimensional measures to assess its implementation. Content validation of the evaluative questions was performed using the Delphi technique. The logical model developed was essential to understand the basic theoretical premises of the PPAHE, condensing the peculiarities of its components into an image and constituting a product that can be used in further evaluative research. The multidimensional instrument presented a content validation index of $91 \%$. The study provides a logical model and a valid multidimensional instrument, which are essential for planning the actual evaluation of the implementation of the PPAHE.
\end{abstract}

Keywords: Nutrition programs and policies; Food and nutrition education; Primary health care; Program evaluation.

\title{
Resumen
}

Las enfermedades crónicas no transmisibles (ENT) aparecen en todo el mundo como un importante problema de salud pública y una nutrición inadecuada es uno de sus principales determinantes. Por tanto, la cualificación de los profesionales sanitarios para llevar a cabo acciones de promoción de una alimentación adecuada y saludable, que sean prácticas, eficaces y viables, es fundamental. El objetivo de este artículo fue realizar el estudio de evaluabilidad, considerando la descripción de la intervención (implementación del Programa de Promoción de la Alimentación Adecuada y Saludable - PPAAS en la Atención Primaria de Salud Brasileña), la elaboración del modelo lógico y la identificación y validación de las preguntas evaluativas. Se adoptó como referencia el sistema de siete elementos propuesto por Thurston y Ramaliu. Se realizaron análisis de documentos, revisión teórica, reuniones para la elaboración del modelo lógico teórico del Programa y la identificación de preguntas evaluativas, lo que resultó en el instrumento de medidas multidimensionales para evaluar su implementación. La validación de contenido de las preguntas evaluativas se realizó mediante la técnica Delphi. El modelo lógico desarrollado fue fundamental para compreender las premisas teóricas básicas del PPAAS, condensando las peculiaridades de sus componentes en una imagen y constituyendo un producto que pueda ser utilizado en futuras investigaciones evaluativas. El instrumento multidimensional presentó un índice de validación de contenido del 91\%. El estudio proporciona un modelo lógico y un instrumento multidimensional válido, que son fundamentales para planificar la evaluación real de la implementación del PPAAS.

Palabras clave: Programas y políticas de nutrición y alimentación; Educación alimentaria y nutricional; Atención primaria de salud; Evaluación de programas y proyectos de salud.

\section{Introdução}

As doenças crônicas não transmissíveis (DCNT) e seus fatores de risco são a principal causa de morbidade, mortalidade e incapacidade no mundo, e constituem um desafio complexo em saúde pública, além de grave ameaça ao desenvolvimento econômico e social. As DCNT constituem sete das 10 principais causas de morte no mundo, de acordo com as Estimativas Globais de Saúde de 2019, sendo responsáveis por 44\% de todas as mortes ou $80 \%$ das 10 entre 2000 a 2019. No ano de 2019, todas as DCNT juntas foram responsáveis por 74\% das mortes em todo o mundo (Malta et al., 2019; World Health Organization [WHO], 2020). A carga global dos principais fatores de risco para morte e anos de vida perdidos por incapacidade em 2019 para adultos e idosos incluíam elevados níveis de pressão arterial, de glicemia, de colesterol LDL e de peso, fatores de risco para DCNT como Diabetes Mellitus (DM) e Hipertensão Arterial Sistêmica (HAS; Global Burden Disease [GBD], 2020).

Na região das Américas, as DCNT são responsáveis por 80,7\% de todas as mortes na região. Desse número total de mortes por DCNT, 38,9\% são mortes prematuras ocorridas em pessoas com menos de 70 anos de idade (Organización Panamericana de la Salud [OPS], 2019). No Brasil, as doenças crônicas não transmissíveis (DCNT) constituem o problema de saúde de maior magnitude e correspondem a 76\% das causas de mortes (Ministério da Saúde [MS], 2011), Malta et al, 2017).

Esse cenário exige o desenvolvimento de políticas que subsidiem ações de saúde diferenciadas que possam garantir, além das ações assistenciais, aquelas direcionadas à educação em saúde e a qualificação profissional com vistas a oportunizar 
modos saudáveis de viver à população e redução da morbimortalidade, sobretudo relacionada à alimentação (Coutinho et al., 2008; Mendes, 2011).

A alimentação é um dos principais determinantes da saúde dos indivíduos (Malta et al., 2019). No entanto, as transformações sofridas no padrão alimentar, nas últimas décadas, como a redução do consumo de alimentos in natura e o concomitante ao aumento dos alimentos ultraprocessados, agravaram o cenário da saúde pública atual e favoreceram a ocorrência das doenças crônicas não transmissíveis (DCNT; MS, 2014). Para deter o avanço das DCNT, são necessários esforços que incluem ações de promoção da alimentação adequada e saudável, de caráter individual e coletivo, pautadas na promoção à saúde, sobretudo focadas na Atenção Primária à Saúde (APS; MS, 2011, 2012).

É amplamente conhecida e discutida, no meio acadêmico, a grande transformação epidemiológica da população, que, nas últimas décadas, experimentou mudanças no seu padrão de saúde e consumo alimentar. Essas transformações acompanharam a redução da pobreza, da fome e da desnutrição. Contudo, observa-se um aumento dos problemas relacionados à Alimentação e Nutrição (AN), caracterizados pelo avanço e coexistência da má nutrição em todas as suas formas, incluindo a desnutrição, a obesidade e outros riscos alimentares para Doenças Crônicas Não Transmissíveis (DCNT), que compartilham determinantes em comum (Swinburn et al, 2019). A má nutrição em todas as suas formas, incluindo a desnutrição, a obesidade e outros riscos alimentares para doenças crônicas não transmissíveis (DCNT). Então, essa má nutrição é, de longe, a principal causa (19\%) de doenças e mortes prematuras no mundo todo (Swinburn et al, 2019).

No Brasil a promoção da alimentação adequada e saudável é uma das diretrizes da Política Nacional de Alimentação e Nutrição (MS, 2012) e um dos temas prioritários da Política Nacional de Promoção da Saúde (MS, 2010). Diante disso, o Ministério da Saúde brasileiro tem envidado esforços para o desenvolvimento de diretrizes nacionais, materiais instrucionais e atividades de qualificação profissional sobre a temática visando capacitar as equipes de saúde para atuação prática. A Promoção da Alimentação Adequada e Saudável (PAAS) compreende um conjunto de estratégias que proporcionem aos indivíduos e coletividades a realização de práticas alimentares apropriadas, harmônicas e em consonância com o uso sustentável do meio ambiente. A alimentação adequada e saudável (a qual se refere à diretriz) pode ser entendida como a oferta de alimento de qualidade, em quantidade necessária, acessível financeiramente e fisicamente, contribuindo, assim, com a queda do número de indivíduos com excesso de peso e comportamentos de risco para as DCNT (Canella et al, 2013).

Nesse cenário, é que surgiu o Instrutivo: Metodologias de Trabalho em Grupo para Promoção de Ações de Alimentação Adequada e Saudável na Atenção Básica (MS, 2016a), o primeiro desdobramento do Guia Alimentar para a População Brasileira (MS, 2014), com o objetivo de apoiar o planejamento e o desenvolvimento de ações coletivas de promoção da alimentação adequada e saudável na APS. Para isso, ele apresenta uma proposta de metodologia de grupo, com suporte teórico e prático para o desenvolvimento das ações (MS, 2016a), que tem como desdobramento o Programa de Promoção da Alimentação Adequada e Saudável (PPAAS) que pode ser desenvolvido por profissionais de saúde em seus lócus de trabalho.

Dada à importância da alimentação adequada e saudável para a promoção da saúde, a prevenção e o controle das DCNT, o PPAAS foi desenvolvido como uma forma de intervenção para apoiar o planejamento e o desenvolvimento de ações coletivas de promoção da alimentação adequada e saudável na atenção primária, com propostas de metodologias ativas e suporte teórico e prático para o desenvolvimento das ações que podem ser realizadas por todos profissionais de saúde (BRASIL, 2016a). Estratégias inovadoras devem ser buscadas a fim de incitar mudança nas práticas das ações coletivas de promoção da alimentação adequada e saudável. A adoção de metodologias não convencionais, pautadas em modelos de ensino-aprendizagem integradores e participativos, a exemplo das metodologias ativas, pode ser útil no processo de promoção de saúde (Menezes et al, 2015; Souza et al, 2018; Alvarez \& Moya, 2020). 
O PPAAS foi implantado a partir de 2017 no Estado de Minas Gerais, com base no instrutivo Metodologias de Trabalho em Grupo para Promoção de Ações de Alimentação Adequada e Saudável na Atenção Básica (MS, 2016a) e pretende ser um orientador da mudança de prática no que se refere à abordagem da promoção da saúde na APS. A metodologia apresentada neste Instrutivo engloba o uso de estratégias e materiais educativos, que objetivam promover encontros dinâmicos, participativos e compreensíveis para os diferentes ciclos da vida.

O PPAAS, aqui analisado, pretende ser um orientador da mudança de prática no que se refere à abordagem da promoção da saúde ao apoiar os profissionais da APS a realizarem ações de promoção da alimentação adequada e saudável práticas e efetivas, além de viáveis em relação ao tempo e infraestrutura disponíveis. As ações do Programa são voltadas para adultos e idosos; entretanto, podem ser adaptadas aos diferentes ciclos da vida e condições de saúde, de acordo com a realidade local. A metodologia de grupo apresentada engloba o uso de estratégias e materiais educativos, que objetivam promover encontros dinâmicos, participativos e compreensíveis para usuários com diferentes graus de instrução (MS, 2016a).

No entanto, não basta dispor de um Programa, é necessário avaliar a sua implantação visando subsidiar o desempenho da intervenção proposta e a otimização dos processos de trabalho no planejamento e execução das ações educativas e de seus resultados. Para isso, a pesquisa avaliativa pode contribuir para a identificação de problemas relacionados à organização e funcionamento de Programas e sustentar decisões direcionadas ao seu aprimoramento e consolidação (Champagne, Brouselle, Hartz, Contandriopoulos\& Denis, 2011/2016). Dentre as abordagens avaliativas, a análise lógica, denominada também de estudo de avaliabilidade (EA), tem sido utilizada para verificar em que medida uma intervenção pode ser avaliada. O EA é um tipo de estudo que antecede a avaliação propriamente dita e tem por finalidade descrever, de forma coerente, um plano de avaliação, tornando-o mais consistente e confiável, além de favorecer a racionalização de recursos financeiros e logísticos (Thurston \& Ramaliu, 2005). Este tipo de abordagem avaliativa, também denominado evaluability assessment (estudo da avaliabilidade - EA) traduz em um conjunto de procedimentos que objetiva o planejamento de avaliações de programas. Os produtos almejados neste estudo incluem a descrição completa do programa, a definição das questões coerentes a serem abordadas na avaliação, um plano para o desenvolvimento da avaliação e um acordo entre as partes interessadas. Esta é uma importante estratégia para o planejamento de avaliações subsequentes maximizando seus potenciais (Trevisan \& Huang, 2003).

Nesse contexto, a pesquisa avaliativa vem se destacando, já que o conhecimento gerado nesse tipo de investigação tem a propriedade de contribuir para a identificação de problemas relacionados à organização e funcionamento de programas e sustentar decisões direcionadas ao seu aprimoramento e consolidação (Champagne, Brouselle, Hartz, Contandriopoulos\& Denis, 2016).

Com a operacionalização do PPAAS, identificou-se a necessidade de avaliar a implantação (Champagne, Brouselle, Hartz, Contandriopoulos\& Denis, 2011/2016) desse programa, por ser uma experiência inovadora na otimização do processo de trabalho no planejamento e execução das ações educativas; melhor qualidade e acesso das ações educativas sobre alimentação adequada e saudável; aumento da motivação das pessoas para mudança de hábitos alimentares e estado nutricional, e haver necessidade de acompanhar a implantação e a reorganização que esse programa pode ou não alcançar. Desta forma, o objetivo deste estudo foi realizar o estudo de avaliabilidade, considerando a descrição da intervenção (implantação do PPAAS na APS), a elaboração do modelo lógico e a identificação e validação das questões avaliativas.

\section{Método}

O referencial teórico metodológico utilizado para realizar o estudo de avaliabilidade foi o sistema dos sete elementos, proposto por Thurston e Ramaliu (2005) que inclui: 1) descrição do programa, identificando suas metas, objetivos e atividades; 2) identificação e revisão dos documentos disponíveis no programa; 3) modelagem dos recursos disponíveis, das atividades pretendidas, impactos esperados e conexões causais presumidas (Modelo Lógico do Programa); 4) supervisão do programa, ou 
obtenção de um entendimento preliminar de como opera; 5) desenvolvimento de um Modelo Teórico da Avaliação; 6) identificação de usuários da avaliação e outros principais envolvidos; 7) obtenção de um acordo quanto ao procedimento de uma avaliação. Esses estudos são relevantes e podem agregar à comunidade científica, novos referenciais teóricos e metodológicos na avaliação de serviços, programas e práticas de saúde (Baratiere et al., 2019).

Foram analisados documentos incluindo manuais, portarias, normas técnicas, guidelines, além de artigos disponíveis nas bases de dados PubMed, SciELO, Lilacs e Medline. Concomitantemente, foram realizadas seis reuniões de trabalho, que resultaram em discussões amplas sobre a implantação do PPAAS na Atenção Primária à Saúde.

Com as informações obtidas nas reuniões, juntamente com a revisão teórica e análises de documentos, foi possível construir o modelo lógico do Programa na perspectiva dos autores Champagne, Brouselle, Hartz e Contandriopoulos (2011/2016) e a obtenção de um acordo quanto ao procedimento do EA. O modelo lógico possibilita explicitar os vínculos com frequência complexos que se articulam no curso do tempo entre uma intervenção (estruturas e processos) e seus efeitos e permite documentar o sentido de um programa graças à conceitualização dos vínculos entre as estruturas, os processos e os resultados (Champagne, Brouselle, Hartz \&Contandriopoulos, 2011/2016).

O modelo lógico possibilitou identificar 42 questões avaliativas relacionadas às dimensões de estrutura $(n=22)$ e processo $(n=20)$ para compor o instrumento de medida (matriz de análise e julgamento) a ser utilizado na avaliação da implantação do PPAAS. Utilizou-se a técnica de Delfos (Delphi) para a validação de conteúdo dessas questões (Pereira \& Alvin, 2015; Scarparo et al, 2012).

A técnica Delphi constitui um método de pesquisa importante no qual se busca, de maneira sistematizada, um consenso de opiniões de um grupo de especialistas a respeito de um problema complexo, quando não existem ainda instrumentos padronizados para sua avaliação (Jünger et al., 2017). Neste estudo, foram convidados a participar do painel, 11 juízas com experiência nos campos de nutrição e saúde pública, dentre elas: duas com pós-doutorado, cinco com doutorado, três com mestrado e uma com pós-graduação. Haynes et al (1995) sugerem que um número de seis a 20 juízes participem do processo de validação. As juízas foram selecionadas por conveniência mediante os seguintes critérios: (i) conhecer o PPAAS, (ii) ter experiência como nutricionista da rede do Sistema Único de Saúde e/ou (iii) ter experiência em pesquisa na temática da educação alimentar e nutricional no SUS.

Foi enviado, por meio do software Google Forms $®$, formulário que continham questões elaboradas e solicitava que cada uma delas ponderasse as perguntas quanto aos critérios propostos por (Pasquali, 2010): relevância, objetividade e clareza. As respostas foram organizadas em uma escala Likert - CP: concordo plenamente; C: concordo; NN: não discordo nem concordo; D: discordo; DT: discordo totalmente. Além disso, cada questão contava com um espaço para que as juízas sugerissem alterações ou fizessem comentários (Pasquali, 2010). Essa técnica de levantamento utilizando um comitê de juízes por e-mail ou Internet é um procedimento relativamente recente, que possui vantagens como a rapidez na distribuição dos questionários e a coleta e o processamento de dados são feitos rapidamente. Para o respondente, há a facilidade de responder às questões quando julgar pertinente e sem a necessidade de um encontro presencial (Timmins, 2015).

Para análise dos dados, utilizou-se o Índice de Validade de Conteúdo (IVC) para identificar o grau de concordância dos especialistas. A indicação de ponto de corte para obtenção do consenso varia na literatura entre os valores de 50 a $80 \%$ (Jünger et al., 2017), tendo sido escolhido o ponto de corte de 70\% de concordância neste estudo, ou seja, IVC igual ou maior 0,70. Se este consenso não é alcançado na primeira rodada, define-se que sejam feitas até três rodadas do questionário entre os participantes. O IVC foi calculado a partir da somatória de respostas classificadas como concordância (CP e C) de cada item do questionário, dividida pelo número total de respostas e multiplicado por 100 (IVC = número de resposta $\mathrm{CP}+\mathrm{C} /$ número total de respostas x 100). Calcularam-se também os IVC médios dos critérios para cada item, somando-se cada percentual obtido em 
cada critério e dividindo-os por três, ou seja, pelos três critérios utilizados. O IVC varia entre 0 e 1 e, quanto mais próximo de 1, melhor será o desempenho do item segundo os juízes (Pereira \&Alvim, 2015).

As questões avaliativas validadas compuseram as matrizes de análise que serão utilizadas para avaliar o grau de implantação (GI) do PPAAS em pesquisa futura.

Este EA faz parte de uma pesquisa maior intitulada Avaliação da Implantação e da Efetividade do Programa de Promoção da Alimentação Adequada e Saudável na Atenção Primária à Saúde, que tem como objetivo geral avaliar a implantação e a efetividade do PPAAS e foi aprovada pelo Comitê de Ética da Universidade Federal de Minas Gerais sob $n^{\circ}$ CAAE 56698716.2.0000.5149.

\section{Resultados}

Inicialmente, foram realizadas a revisão da literatura e a análise de documentos oficiais para melhor compreensão da proposta da avaliação do PPAAS, dentre eles: Política Nacional de Promoção da Saúde (MS, 2010); Plano de Ações Estratégicas para o Enfrentamento das Doenças Crônicas Não Transmissíveis no Brasil 2011-2022 (MS, 2011); Política Nacional de Atenção Básica (MS, 2012); Marco de Referência de Educação Alimentar e Nutricional para as Políticas Públicas (Ministério do Desenvolvimento Social e Combate à Fome [MDS], 2012); Política Nacional de Alimentação e Nutrição (MS, 2013); Estratégia Intersetorial de Prevenção e Controle da Obesidade (Câmara Interministerial de Segurança Alimentar e Nutricional [CAISAN], 2014); Guia Alimentar para População Brasileira (MS, 2014) e Instrutivo de Metodologias de Trabalho em Grupo para Promoção de Ações de Alimentação Adequada e Saudável na Atenção Básica (MS, 2016a).

Na Figura 1 está representada uma linha do tempo em que é possível observar alguns marcos da Promoção da Alimentação Adequada e Saudável no Brasil e as estratégias adotadas para a prevenção das DCNT no país. O Instrutivo Metodologias de Trabalho em Grupo para Promoção de Ações de Alimentação Adequada e Saudável na Atenção Básica que embasa o PPAAS é um desdobramento da $2^{\mathrm{a}}$ Edição do Guia Alimentar para a População Brasileira publicado em 2014 (MS, 2014). 
Figura 1: Linha do tempo dos marcos da Promoção da Alimentação Saudável e estratégias para prevenção das Doenças Crônicas Não Transmissíveis no Brasil.

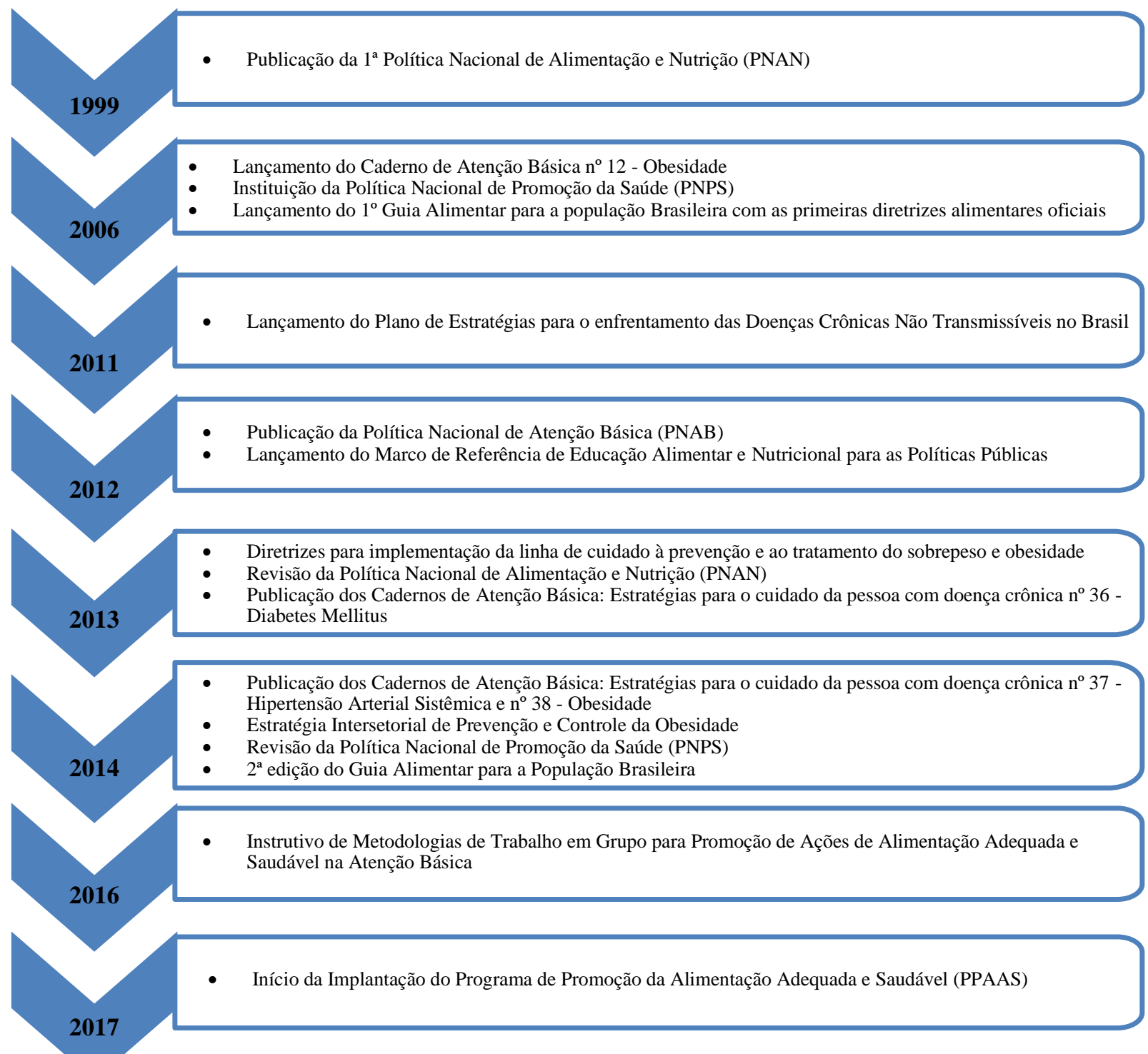

Fonte: Autores (2021).

O PPAAS é baseado na realização das ações educativas previstas no Instrutivo: Metodologia de trabalho em grupos para ações de alimentação e nutrição na Atenção Básica (MS, 2016a). Para sua implantação é realizada previamente qualificação dos profissionais de saúde por meio de Educação Permanente sobre Metodologias de Trabalho em Grupo para a Promoção da Alimentação Adequada e Saudável. Essa atividade é realizada em quatro encontros com carga horária total de 16 horas e disponibilizada Tutoria Virtual realizada por meio do aplicativo Whatsapp ${ }^{\circledR}$

As ações educativas propostas são referenciadas em literatura sobre DCNT, educação em saúde, educação alimentar e nutricional e nutricional, promoção da alimentação adequada e saudável, e abordagem coletiva das ações de educação alimentar e nutricional na APS apresentada no Instrutivo Metodologia de trabalho em grupos para ações de alimentação e nutrição na Atenção Básica (MS, 2016a), que descreve ainda as atividades propostas detalhadamente, incluindo um roteiro para desenvolvimento, com grau de dificuldade para realização, objetivos, sugestão de categorias profissionais envolvidas, tempo de duração, materiais necessários para aplicação da atividade, referencial teórico, imagens e fotos ilustrativas. A metodologia apresentada no Instrutivo engloba o uso de estratégias e materiais educativos como oficinas, ações no ambiente, 
painéis, diário de bordo, folders, uso de livro de receitas Na cozinha com frutas, verduras e legumes (MS, 2016b) e livro Desmistificando dúvidas sobre alimentação e nutrição (MS, 2016c), que objetivam promover encontros dinâmicos, participativos e compreensíveis para usuários com diferentes graus de instrução.

Em relação aos elementos 6 e 7 nomeados por Thurston e Ramaliu (2005), este estudo foi proposto e conduzido por pesquisadores da Universidade Federal de Minas Gerais (UFMG), Universidade Federal de São João Del Rei (UFSJ) e Universidade Federal de Ouro Preto (UFOP) e teve adesão de 10 profissionais engajados em diferentes níveis de atuação no PPAAS, incluindo profissionais da rede municipal da APS (stakeholders). Ademais, houve reconhecimento de outros interessados na avaliação, como os gestores da APS, outros profissionais da APS (enfermeiros, psicólogos, nutricionistas e agentes comunitários de saúde, por exemplo) e usuários do Programa.

Para o desenvolvimento do modelo lógico do PPAAS, foram fundamentais a descrição e a compreensão da organização do Programa, dos contextos que o envolvem, e dos componentes considerados essenciais para o sucesso da sua implantação na APS. A pactuação do modelo lógico com os interessados na avaliação, coerente com a revisão teórica e normativa realizada, representou um passo importante para a legitimação do processo avaliativo. A elaboração do modelo lógico do PPAAS possibilitou identificar questões avaliativas para compor a matriz de análise e julgamento, instrumento de medidas a ser utilizado em pesquisa futura sobre avaliação da implantação do PPAAS.

O modelo lógico do PPAAS possibilitou explicitar os vínculos que devem se articular entre as estruturas, processos e resultados que compõem a intervenção (Champagne, Brouselle, Hartz \& Contandriopoulos, 2011/2016). Foram definidos no modelo, quatro componentes estruturais (recursos físicos, materiais, humanos e normatização) e quatro componentes da dimensão processo, foram eles: educação permanente e tutoria, planejamento das atividades, execução das atividades e preenchimento dos instrumentos. Para cada um dos componentes da dimensão processo foram descritas as atividades (serviços ou bens produzidos) e os resultados esperados em curto, médio e longo prazo (impacto), conforme mostrado na Figura 2. 
Research, Society and Development, v. 10, n. 10, e600101019051, 2021

(CC BY 4.0) | ISSN 2525-3409 | DOI: http://dx.doi.org/10.33448/rsd-v10i10.19051

Figura 2. Modelo lógico do Programa de Promoção da Alimentação Adequada e Saudável na Atenção Primária à Saúde.

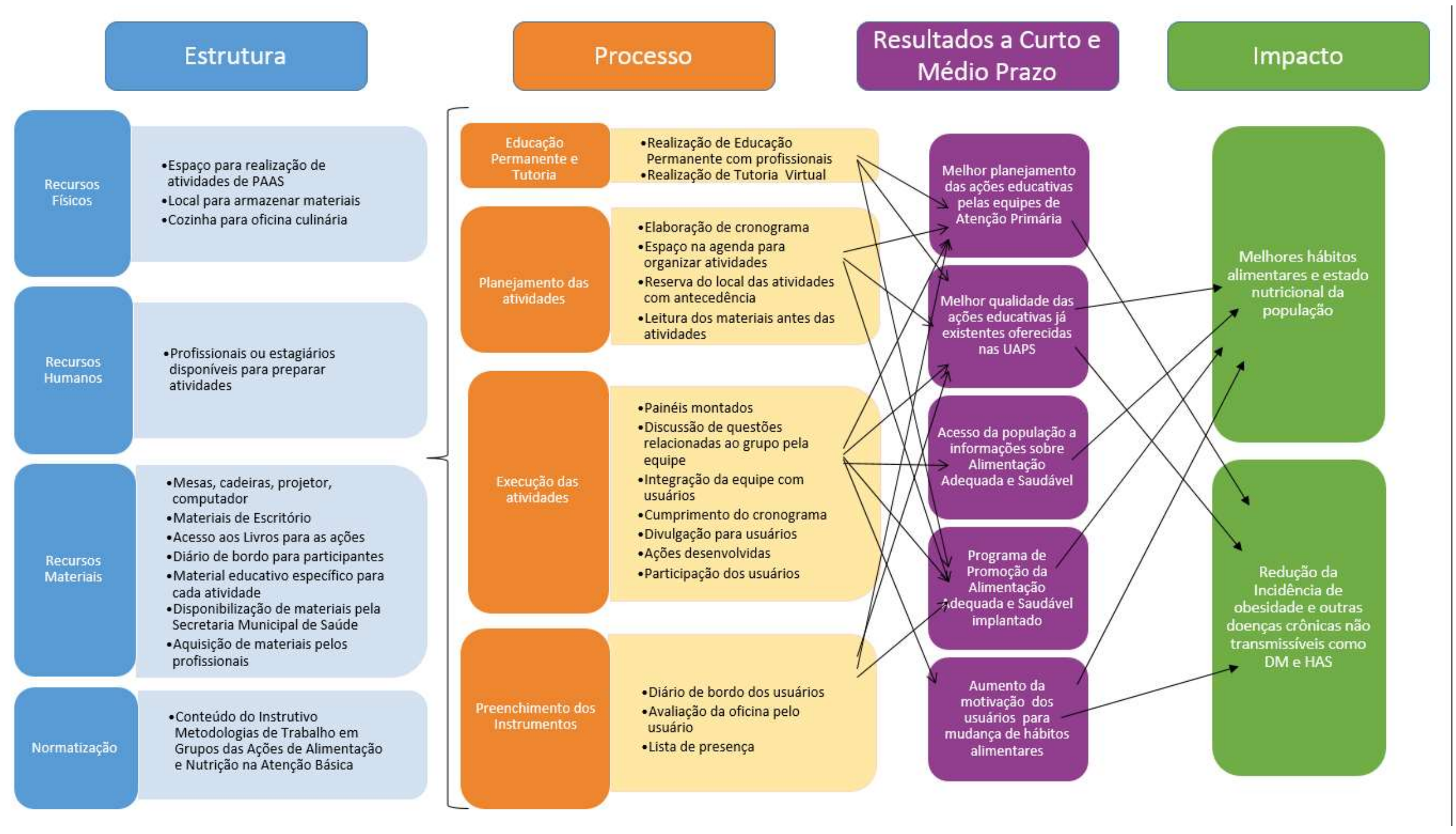

Fonte: Autores (2021). 
Research, Society and Development, v. 10, n. 10, e600101019051, 2021

(CC BY 4.0) | ISSN 2525-3409 | DOI: http://dx.doi.org/10.33448/rsd-v10i10.19051

O desenvolvimento do modelo lógico garantiu a identificação dos componentes a serem avaliados e as relações causais presumidas, além da identificação das 42 questões avaliativas segundo as dimensões de estrutura ( $\mathrm{n}=22$ ) e processo $(n=20)$, que comporão a matriz de análise e julgamento (instrumento de medidas para avaliar a implantação do PPAAS).

Para a validação de conteúdo das questões avaliativas, sete juízas (64\%) responderam o formulário, sendo 71,4\% pesquisadoras e 28,6\% profissionais da rede do Sistema Único de Saúde. De acordo com o grau de formação, 14,3\% tem pósdoutorado, 42,9\% doutorado, 28,6\% mestrado e 14,3\% pós-graduação latu sensu. Considerando as duas dimensões (estrutura e processo), o IVC total foi de $91 \%$. Obteve-se na dimensão estrutura um IVC médio de 88,0\% e 95,0\% \% na dimensão processo. Segundo os componentes do modelo lógico, observaram-se IVC médio de 94,0\% para o componente Recursos Físicos; 95,0\% para Recursos Humanos; 86,0\% para Recursos Materiais; 95,0\% para Normatização; 95,0\% para Educação Permanente e Tutoria Virtual; 97,0\% para Planejamento da atividades educativas do PPAAS; 93,0\% para Execução das atividades educativas e 93,0\% para Preenchimento dos instrumentos do PPAAS. De forma geral, os juízes relataram que as questões são claras e de fácil compreensão.

As questões validadas compuseram a matriz de análise e julgamento, cuja finalidade é realizar, em pesquisa futura, a avaliação do grau de implantação do PPAAS e com isso, identificar as suas potencialidades e fragilidades. Nessa matriz foram apresentados para cada questão, das dimensões estrutura e processo, os critérios, o tipo de abordagem, a fonte de dados, a técnica de coleta, a descrição e a pontuação máxima, que variou de 5 a 15 pontos (Figuras 3 e 4). 
Research, Society and Development, v. 10, n. 10, e600101019051, 2021

(CC BY 4.0) | ISSN 2525-3409 | DOI: http://dx.doi.org/10.33448/rsd-v10i10.19051

Figura 3. Matriz de análise e julgamento para avaliar a Dimensão Estrutura da implantação do Programa de Promoção da Alimentação Adequada e Saudável.

\begin{tabular}{|c|c|c|c|c|c|c|}
\hline $\begin{array}{l}\text { DIMENSAO: } \\
\text { ESTRUTURA }\end{array}$ & CRITÉRIOS & $\begin{array}{c}\text { TIPO DE } \\
\text { ABORDAGEM }\end{array}$ & $\begin{array}{c}\text { FONTE DE } \\
\text { DADOS }\end{array}$ & TÉCNICA DE COLETA & DESCRIÇÁAO & $\begin{array}{c}\text { PONTUACÁRO } \\
\text { MAXIMA }\end{array}$ \\
\hline \multirow{3}{*}{ 1. Recursos Físicos } & Espaça/ssala para realizaç̧ăo dass ativididades & Quantitativa & Primário & Questionisio & 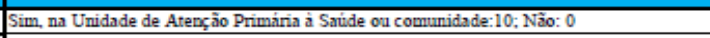 & 10 \\
\hline & 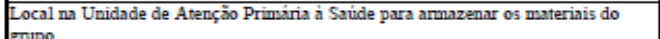 & Quantitativa & Primánio & Questionírio & Sim: 10: Parcialmente - guardou em outro lugar, como em casal fora da unidade: 5 ; Não: 0 & 10 \\
\hline & 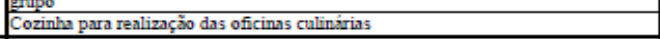 & Quantitativa & Primário & Questionisio & Sim:10: Parcialmente - adapptou espaço ou a oficina- 5: Năo:0 & 10 \\
\hline 2. Recursos Humanos & Profissionais e eou estagiarios disponiveis para preparar as atividades & Quantitativa & Primánio & Questionisio & 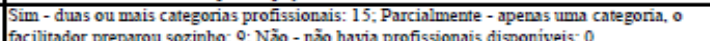 & 15 \\
\hline \multirow{6}{*}{$\begin{array}{l}\text { 3.1. Recursos } \\
\text { Materiai I. Comuns } \\
\text { todas as atividades }\end{array}$} & Finstalasçōes e equipamentos adequados para as atividads & Quantitativa & Primárío & Questionisio & 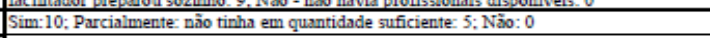 & 10 \\
\hline & 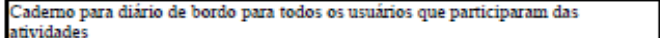 & Quantitativa & Primário & Questionário & Sim:10: Parcialmente - realizou adaptação para que todos tivessem acesso: 5 ; Não: 0 & 10 \\
\hline & 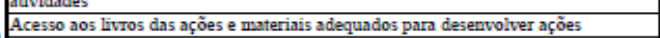 & Quantitativa & Primário & Questionisio & Sim:10; Parcialmente - dificil acesso, adappou a oficina: 5; Năo: 0 & 10 \\
\hline & Materiais educativos preparados previamente & Quantitativa & Primário & Questionisio & Sim:10: Parcialmente - faltaram algums materiais, atraso na disponibibilização: 5; Não: 0 & 10 \\
\hline & Secretaria de saúde disponibilizou materinis de escritónio & Quantitativa & Primário & Questionisio & Sim:10: Paciamente- quantidade insuriciente, qualidade rüm 5; Näo:0 & 10 \\
\hline & 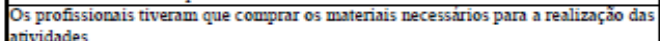 & Quantitativa & Primánio & Questionário & Sim:0; Pacialmente - alguns materiais: 5; Não:10 & 10 \\
\hline \multirow{21}{*}{$\begin{array}{l}\text { 3.2. Recursos } \\
\text { Materiais II- } \\
\text { Especificos para cada } \\
\text { atridade }\end{array}$} & 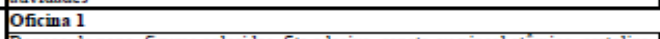 & & & & & \\
\hline & 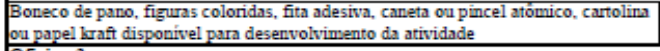 & Quantitativa & Primário & Questionisio & Sim-5; Adagtơ a atividade com itens simulares: 3; Não: 0 & 5 \\
\hline & Oficina 2 & & & & & \\
\hline & 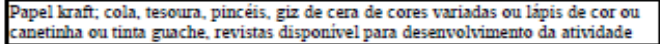 & Quantitativa & Primário & Questionisio & Sim-5; Adaptou a atividade com itens similares: 3; Não: 0 & 5 \\
\hline & Oficina 3 & & & & & \\
\hline & $\begin{array}{l}\text { Caralina ou papel kraft, pincel atoomico disponivel para desenvolvimento da } \\
\text { atavidade }\end{array}$ & Qunntitaiva & Primário & Questionário & Sim-5: Adapptou a aúvidade com itens similalaes: 3 ; Não: 0 & 5 \\
\hline & Diciua 4 4 & & & & & \\
\hline & 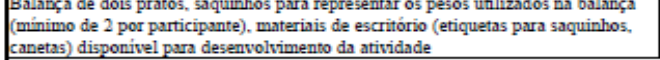 & Quantitativa & Primário & Questionário & Sim-5; Adaptou a atividade com itens similares: 3; Nâo: 0 & 5 \\
\hline & Oficina 5 . & & & & & \\
\hline & 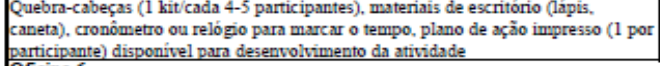 & Quantitativa & Primário & Questionário & Sim-5; Adapptou a atividade com itens simvilares: 3; Nẫ: 0 & 5 \\
\hline & 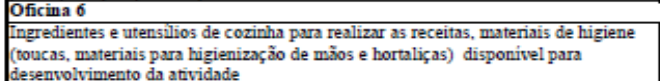 & Quantitativa & Primánio & $\begin{array}{l}\text { Questionário durante a } \\
\text { implantaçáo }\end{array}$ & Sim:-5; Adapptou a atividade com itens similares: 3: Nẫo: 0 & 5 \\
\hline & \begin{tabular}{|l|} 
desenviovimento da atvidade \\
Oficina 7 \\
\end{tabular} & & & & & \\
\hline & 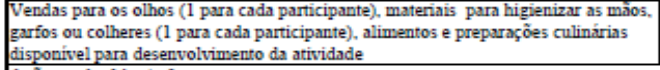 & Quantitativa & Primário & Questionário & Sim-5; Adaptou a atividade com itens simvilares: 3; Nẫo: 0 & 5 \\
\hline & 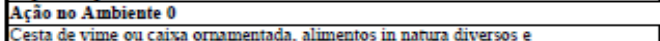 & & & & & \\
\hline & 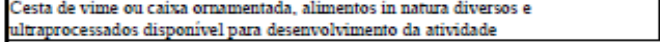 & Qunntitativa & Primário & Questionário & Sim-5; Adaptou a a atividade com itens simuilares: 3; Nâo: 0 & 5 \\
\hline & Açấo no Ambiente 1 & & & & & \\
\hline & Carrinho de supermercado e espelho disponivel para desenvolvimento da atividade & Quantitativa & Primário & Questionário & Sim-5; Adaptou a atividade com itens similares: 3; Não: 0 & 5 \\
\hline & Ação no Ambiente 2 & & & & & \\
\hline & 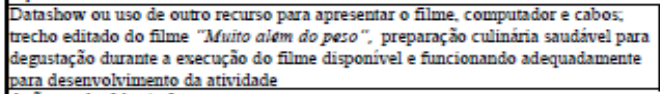 & Quantitativa & Primánio & Questionírio & Sim-5; Adapptou a atividade com itens simuilares: 3: Nẫo: 0 & 5 \\
\hline & 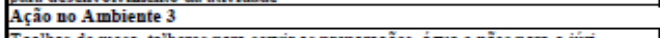 & & & & & \\
\hline & 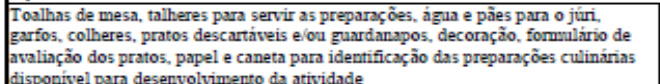 & Quantitativa & Primário & Questionário & Sim-5; Adapptou a atividade com itens simulares: 3: Nẫo: 0 & 5 \\
\hline 4. Normatização & 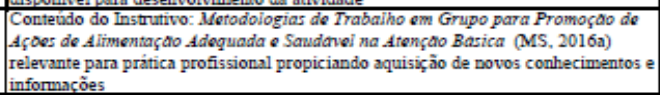 & Quantitativa & Primário & Questionizio & Sim: 10: Parcialmente: $5 ;$ Năo: 0 & 5 \\
\hline
\end{tabular}

Fonte: Autores (2021). 
Research, Society and Development, v. 10, n. 10, e600101019051, 2021

(CC BY 4.0) | ISSN 2525-3409 | DOI: http://dx.doi.org/10.33448/rsd-v10i10.19051

Figura 4. Matriz de análise e julgamento para avaliar a Dimensão Processo da implantação do Programa de Promoção da Alimentação Adequada e Saudável.

\begin{tabular}{|c|c|c|c|c|c|c|}
\hline $\begin{array}{l}\text { DIMENSÃO: } \\
\text { PROCESSO }\end{array}$ & CRITÉRTOS & $\begin{array}{c}\text { TIPO DE } \\
\text { ABORDAGEM }\end{array}$ & $\begin{array}{c}\text { FONIE DE } \\
\text { DADOS }\end{array}$ & TÉCNICA DE COLETA & DESCRIÇÃO & \begin{tabular}{|c|} 
PONTUACCÃO \\
MÁXTMA
\end{tabular} \\
\hline \multirow{2}{*}{$\begin{array}{l}\text { 1. Realizaçāo da } \\
\text { Educação Permanente } \\
\text { sobre o PPAAS e } \\
\text { Tutoria Virtual } \\
\end{array}$} & $\begin{array}{l}\text { Oóerta de ediucaçăo permanente para profisssionais da equipe para unlizaç̧̆o do } \\
\text { manterial do PPAAS }\end{array}$ & Qualitativa & Primánio & Questionário & Sim: 10; Não:0 & 10 \\
\hline & Dispoubbilização de Tutoria virtual para apoio das atividades coletrivas do PPAAS & Qualitativa & Primário & Questionainio & Sim 10; Não:0 & 10 \\
\hline \multirow{7}{*}{$\begin{array}{l}\text { 2. Planejamento das } \\
\text { atividades educativas } \\
\text { do PPAAS }\end{array}$} & Equipe elaborou um cronograma com as datas dos grupos & Quantitativa & Primário & Questionanio & $\operatorname{Sim} 10 ;$ Sim mas com ataaso: $5 ;$ Nà̃o: 0 & 10 \\
\hline & $\begin{array}{l}\text { lequipe reservou espaço na agenda para organizaçaio dos materiais para a conduçáio } \\
\text { do gropo }\end{array}$ & Quantitativa & Primário & Questionánio & Sim-10; Parcialmente: $5 ;$ Não: 0 & 10 \\
\hline & Equipe reservou local para realizar a ação com antecedência & Quantitativa & Primário & Questionário & Sim: 10; Parcialmente: 5; Não: 0 & 10 \\
\hline & \begin{tabular}{|l|} 
Equipe se reumiu e discutuiu questōes relacionadas aos encontros \\
\end{tabular} & Quantitativa & Primário & Questionahio & Sim - sempre ou na maioria das vezes: 10; Algumas vezes: 5 ; Nầ - muluca ou raramente: 0 & 10 \\
\hline & Participação dos outros profissionais na atividade coletiva, além do coordenador & Quantitativa & Primário & Questionario & 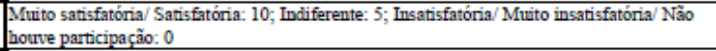 & 10 \\
\hline & $\begin{array}{l}\text { Coordenador do grupo leu o Instrutivo previamente para se preparar para } \\
\text { aplicação da atividade }\end{array}$ & Quantitativa & Primário & Questionário & Sim-10; Pacialmente: $5 ;$ Não:0 & 10 \\
\hline & $\begin{array}{l}\text { A equipe leu o onstrutivo previamente ao dia do grupo e se preparar para aplicaşào } \\
\text { da atrivdade }\end{array}$ & Quantitativa & Primário & Questionánio & Sim: 10; Pacialmente: $5 ;$ Nà̃o:0 & 10 \\
\hline \multirow{8}{*}{$\begin{array}{l}\text { 3. Erecução das } \\
\text { atividades educativas } \\
\text { do PPAAS }\end{array}$} & Quatro painéis foram montados no espaço da umidade de saíde & Quantitativa & Primário & Questionánio & Sim 10; Pacialmente: $5 ;$ Não:0 & 10 \\
\hline & Integração da equipe com usuários & Quantitativa & Primário & Questioninio & 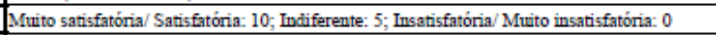 & 10 \\
\hline & Cumprimento do crovograma pela equipe & Quantitativa & Primário & Questionánio & Sim: 10; Pacialmente: 5; Não:0 & 10 \\
\hline & Divulgaģão para usuários & Quantitativa & Primário & Questionanio & Sim: 10; Ditulgạẫo em cima da hora: 5; Não: 0 & 10 \\
\hline & Busca ativa dos usuários & Quantitativa & Primário & Questionario & Sim: 10; Busca tardia: 5 ; Não: 0 & 10 \\
\hline & Atividades coletivas desentrolvidas & Quantitativa & Primário & Questionánio & 80 a $100 \%$ das atividades: 10 ; Entre 60 a $79 \%$ das atividades: 5; $<60 \%$ das atividades: 0 & 10 \\
\hline & Usuários participativos & Quantitativa & Primário & Questioninio & Sim (sempre ou na maioria das vezes ) : 10; Algumas vezes: 5 ; Não (utuca ou raramente): 0 & 10 \\
\hline & Adesão dos partipantes & Quantitativa & Primário & Questioninio & 70 a $100 \%$ dos usuários: $10 ;$ Entre 50 a $69 \%$ do usuários: $5 ;<50 \%$ dos usuários: 0 & 10 \\
\hline \multirow{3}{*}{$\begin{array}{l}\text { 4. Preenchimento dos } \\
\text { instrumentos do } \\
\text { PPAAS }\end{array}$} & Lista de presença dos participantes dos encontros & Qualitativa & Primário & Questionánio & Sim. 10; Pacialmente: 5 ; Nào:0 & 10 \\
\hline & Avaliação da oficina pelos participantes & Quantitativa & Primário & Questionánio & Sim-10; Pacialmente: 5 ; Não:0 & 10 \\
\hline & Diário de bordo dos participantes & Quantitativa & Primário & Questioninio & 70 a 100\% dos usuários: 10; Entre 50 a $69 \%$ do usuários: 5; <50\% dos usuários: 0 & 10 \\
\hline
\end{tabular}

Fonte: Autores (2021). 


\section{Discussão}

Com o desenvolvimento do EA foi possível justificar cientificamente a decisão de avaliar a implantação do PPAAS e, ainda, conhecer a sua organização e o seu funcionamento. A análise de documentos e a realização de reuniões com os interessados foram imprescindíveis para a obtenção de um entendimento preliminar de como o PPAAS se organiza, considerando as metas, objetivos e atividades que o constituem, possibilitando sua descrição. Essa abordagem avaliativa constituiu uma ferramenta útil que proporcionou explorar a teoria, propósitos e objetivos do PPAAS na elaboração de modelos capazes de esclarecer contextos e verificar plausibilidade das relações entre o problema, a sua estrutura, o processo de implantação e os resultados esperados em curto, médio e longo prazos. O estudo possibilitou ainda o desenvolvimento de um plano para avaliações futuras, na medida em que explorou as expectativas e necessidades dos grupos de interesse envolvidos e o grau de organização e implementação da iniciativa (Craig \& Campbell, 2015; Figueiredo et al, 2019).

Considerada uma definição mais completa e atual, para Contandriopoulos (2006) avaliar consiste em 'fazer um julgamento' de valor sobre uma 'intervenção', empregando dispositivos que permitam fornecer 'informações cientificamente válidas e socialmente legítimas' sobre uma intervenção ou qualquer um de seus componentes. A avaliação apresenta-se assim, como um instrumento essencial de apoio à gestão devido a sua capacidade de melhorar a qualidade da tomada de decisão. Nesta direção, diversas pesquisas avaliativas têm sido utilizadas para produzir informações para apoio e orientação da tomada de decisão por profissionais e gestores de saúde (Dias et al, 2018; Silva et al, 2018).

O modelo lógico desenvolvido foi fundamental para compreensão das premissas teóricas do PPAAS, resumindo em um fluxograma as peculiaridades desse programa, permitindo a identificação das questões avaliativas, essenciais para a condução da avaliação, sendo este um produto da pesquisa que poderá ser utilizado em pesquisas avaliativas futuras e para produção de evidências que pautem a construção de políticas públicas. Como destacado por Champagne, Brouselle, Hartz e Contandriopoulos (2011/2016) a modelização permite explicitar o caminho lógico dos atores e revelar os objetivos tornado possível o aprimoramento da inteligibilidade de um sistema complexo.

Ressalta-se que a alimentação adequada e saudável é de interesse dos países no que se refere à saúde, uma vez que a Organização Mundial da Saúde (OMS) recomenda, por meio da Estratégia Global para a Promoção da Alimentação Saudável, Atividade Física e Saúde, que os governos formulem e atualizem periodicamente diretrizes nacionais sobre alimentação e nutrição, levando em conta mudanças nos hábitos alimentares e nas condições de saúde da população e o progresso no conhecimento científico. Essas diretrizes de promoção da alimentação adequada e saudável devem ter o propósito de apoiar a educação alimentar e nutricional e subsidiar políticas e programas nacionais de alimentação e nutrição compreendendo um conjunto de estratégias que objetivam proporcionar aos indivíduos e coletividades a realização de práticas alimentares apropriadas (MS, 2014). Nesse sentido, o Brasil se destaca pela atualização do Guia Alimentar para a População Brasileira (MS, 2014), e seus produtos derivados que visa a sua disseminação, como o Instrutivo Metodologias de Trabalho em Grupo para Promoção de Ações de Alimentação Adequada e Saudável na Atenção Básica (MS, 2016a).

Além da exploração do contexto da intervenção, outro aspecto relevante na avaliação em saúde é identificar o potencial de utilização dos resultados e se há indicativo de sustentabilidade da intervenção proposta (Wholey, 1977). A cooperação ativa dos engajados no processo de implantação de uma intervenção contribui para os primeiros achados do estudo e para a análise e interpretação dos dados do estudo de avaliabilidade, podendo gerar subsídios para a melhoria do Programa (Thurston \& Ramaliu, 2005). Nesse sentido, o EA realizado envolveu membros pesquisadores e profissionais de saúde de municípios do estado de Minas Gerais na construção do modelo teórico-lógico focalizando nos aspectos centrais a serem avaliados. De acordo com Guba e Lincoln (1989), a negociação entre os atores envolvidos nos processos avaliativos é uma importante característica da avaliação de quarta geração, que se contrapõe às gerações anteriores à década de 1980. Pessoas interessadas e engajadas na avaliação constitui-se uma abordagem fundamental para identificar os problemas e explicar o 
objeto avaliado, de modo a favorecer a incorporação dos resultados na tomada de decisão de profissionais e/ou gestores para aperfeiçoar, ampliar ou alterar a intervenção (Tanaka \&Tamaki, 2012).

Para estruturar um processo avaliativo com capacidade de apoiar a tomada de decisão da gestão em saúde e responder às pretensões das partes interessadas, é necessário considerar um conjunto de princípios, tais como: 1) utilidade: garantia da abordagem de questões relevantes; 2) factibilidade e viabilidade: a avaliação deve ter boa relação de custo-benefício; 3 ) propriedade: garantia da ética e 4) precisão: garantia de que os achados possam ser considerados corretos. O envolvimento dos interessados é a forma de garantir que o processo avaliativo seja de qualidade e atenda aos princípios da avaliação, em especial a utilidade e a propriedade (Tanaka \&Tamaki, 2012).

A validação de conteúdo, realizado por meio da técnica Delphi neste estudo, foi realizada por se mostrar essencial ao desenvolver novos instrumentos de medidas, visto que representa o estágio inicial para a associação de conceitos abstratos com indicadores observáveis e mensuráveis (Coluci et al, 2015). A seleção das questões avaliativas é um ponto decisivo no processo de avaliação, uma vez que se as questões não são claras o produto obtido não é uma avaliação, e sim um diagnóstico da situação. A falta de clareza pode levar ao acúmulo de dados e de informações que poderão ser inúteis para a tomada de decisão a fim de modificar determinada situação de saúde da população (Tanaka \&Tamaki, 2012). A validação pelos juízes por meio da técnica Delphi foi essencial para identificar se havia necessidade de ajustes à matriz quanto ao conteúdo ou à forma de apresentação das questões buscando aumentar o poder analítico do instrumento. Dessa forma, pôde-se concluir que as questões avaliativas propostas eram capazes de identificar os aspectos indispensáveis para compreender a implantação do PPAAS nos municípios.

Embora este estudo tenha sido desenvolvido para avaliação da implantação do PPAAS no âmbito de municípios específicos, ele pode ser reprodutível, com adaptações, em outros municípios do país, uma vez que, o modelo lógico de implantação foi construído a partir das normativas nacionais. Além disso, outro ponto forte do EA é que o próprio envolvimento dos interessados na validação das questões avaliativas possibilita a identificação das potencialidades e fragilidades do Programa, bem como o reconhecimento de suas responsabilidades no processo da avaliação. E ainda, o caráter inédito deste estudo, visto que avalia um programa baseado no Instrutivo de Metodologias de Trabalho em Grupo para Promoção de Ações de Alimentação Adequada e Saudável na Atenção Básica (MS, 2016a) que é o primeiro desdobramento no país da publicação Guia Alimentar para População Brasileira (MS, 2014).

Contudo, destaca-se algumas de suas limitações, entre elas a utilização de questionários por meio eletrônico para realizar a técnica Delphi. Este é ainda um procedimento recente que, apesar de possuir vantagens como rapidez na distribuição, na coleta e no processamento de dados e alcance de atores diversos e potenciais respondentes que podem estar distantes geograficamente, pode levar ao aumento das taxas de não respondentes, o que causa perdas amostrais (Jünger et al., 2017).

O EA confirmou que é possível a realização de uma avaliação sistemática de caráter mais extenso. Além disso, com o envolvimento dos atores interessados, foram elaboradas questões avaliativas adequadas para a avaliação da implantação do PPAAS, a ser realizada no futuro. A construção do plano de avaliação fortalece o poder da ferramenta de avaliação e a utilização de seus achados pela gestão.

\section{Considerações Finais}

O estudo realizado abrangeu a análise das políticas de promoção à saúde e de alimentação e nutrição que culminou na construção do PPAAS, a descrição da intervenção, a modelagem, o planejamento e a construção e validação do instrumento com questões para avaliar o grau e o contexto de sua implantação.

Estudos como este permitem investigar aspectos além da cobertura e efetividade do Programa e podem ser capazes de elucidar a relação entre as dimensões com o contexto da sua implantação. Desta forma, recomenda-se que pesquisadores 
interessados em investigar ações de promoção da alimentação saudável realizem EA como este para garantir que a avaliação proposta tenha capacidade de esclarecer a efetividade das ações, apontando fragilidades e fortalezas.

Pretende-se que o presente estudo contribua para produzir conhecimento de forma sistemática e gerar arcabouço teórico para construção de metodologias de pesquisa de avaliação de implantação de programa de promoção de alimentação saudável para trabalhos futuros, principalmente na Atenção Primária à Saúde, e propor indicadores que contribuam para uma melhor estruturação da avaliação de ações educativas e para produção de evidências científicas que pautem a construção de políticas públicas.

\section{Fontes de Financiamento}

Conselho Nacional de Desenvolvimento Científico e Tecnológico (CNPq) pela bolsa de produtividade de ACSL; Fundação de Amparo à Pesquisa do Estado de Minas Gerais (FAPEMIG) pelo financiamento do projeto (PPSUS CDS - APQ-03720-17); Coordenação de Aperfeiçoamento de Pessoal de Nível Superior - CAPES (Código de Financiamento 001), pelas bolsas de estudo.

\section{Referências}

Alvarez, L. N. R. \& Moya, J. L. M. (2017). El prácticum: eje formador de la práctica reflexiva en enfermeira. Haciapromoc Salud, 22(1),70-83. http://dx.doi.org/10.17151/hpsal.2017.22.1.6

Baratieri, T., Nicolotti, C. A., Natal, S. \& Lacerda, J. T. (2019). Aplicação do Estudo de Avaliabilidade na área da saúde: uma revisão integrativa. Saúde Debate, 43(120), 240-255. https://doi.org/10.1590/0103-1104201912018

Câmara Interministerial de Segurança Alimentar e Nutricional (2014). Estratégia Intersetorial de Prevenção e Controle da Obesidade: recomendações para estados e municípios. CAISAN. https://www.mds.gov.br/webarquivos/publicacao/seguranca_alimentar/estrategia_prevencao_obesidade.pdf

Canella, D. S., Silva, A. C. F. \& Jaime, P. C. (2013). Produção científica sobre nutrição no âmbito da Atenção Primária à Saúde no Brasil: uma revisão de literatura. Ciência \& Saúde Coletiva, 18(2), 297-308. http://www.cienciaesaudecoletiva.com.br/artigos/producao-cientifica-sobre-nutricao-no-ambito-daatencao-primaria-a-saude-no-brasil-uma-revisao-de-literatura/9987

Champagne, F., Brouselle A., Hartz, Z., Contandriopoulos, A. P. \& Denis, J. L. (2016). A Análise da Implantação. In: Brousselle, A., Champagne. F., Contandriopoulos, A. P. \& Hartz, Z. (Org.), Avaliação em saúde: conceitos e métodos (pp.217-238). Editora Fiocruz. $2^{\mathrm{a}}$ reimpressão. (Trabalho original publicado em 2011)

Champagne F., Brouselle, A., Hartz, Z. \& Contandriopoulos, A. P. (2016). Modelizar as Intervenções. In: Brousselle, A., Champagne. F., Contandriopoulos, A. P. \& Hartz, Z. (Org.), Avaliação em saúde: conceitos e métodos (pp.41-60). Editora Fiocruz. $2^{\mathrm{a}}$ reimpressão. (Trabalho original publicado em 2011)

Coluci, M. Z. O, Alexandre, N. M. C. \& Milani, D. (2015). Construção de instrumentos de medida na área da saúde. Ciência \& Saúde Coletiva, 20(3), 925936. https://www.cienciaesaudecoletiva.com.br/artigos/construcao-de-instrumentos-de-medida-na-area-da-saude/12607

Contandriopoulos, A.P. (2006). Avaliando a institucionalização da avaliação. Ciências e Saúde Coletiva, 11(3), 705-711. https://doi.org/10.1590/S141381232006000300017

Coutinho, J. G., Gentil, P. C. \&Toral, N. (2008). A desnutrição e a obesidade no Brasil: o enfrentamento com base na agenda única de nutrição. Caderno de Saúde Pública, 24(2), 332-340. https://doi.org/10.1590/S0102-311X2008001400018

Craig, P., Campbell, M. (2015). Evaluability Assessment: a systematic approach to deciding whether and how to evaluate programmes and policies: a What Works Scotland Working Paper. http://dx.doi.org/10.13140/RG.2.1.2007.4725

Dias, M. S. A., Oliveira, I. P., Silva, L. M. S., Vasconcelos, M. I. O, Franklin, M. F. A. S. M., Forte, D. S. \& Silva, L. C. C. (2018). Política Nacional de Promoção da Saúde: um estudo de avaliabilidade em uma região de saúde no Brasil. Ciências e Saúde Coletiva, 23 (1), 103-114. https://doi.org/10.1590/141381232018231.24682015

Figueiredo, T. A., Angulo-Tuesta, A. \& Hartz, Z. (2019). Avaliabilidade da Política Nacional de Regulação no SUS: uma proposta preliminar. Physis: Revista de Saúde Coletiva, 29(2), 1-26. https://doi.org/10.1590/s0103-73312019290215

Global Burden of Disease. (2020). Global burden of 87 risk factors in 204 countries and territories, 1990-2019: a systematic analysis for the Global Burden of Disease Study 2019, The Lancet, 396, 1223-124. https://www.thelancet.com/action/showPdf?pii=S0140-6736\%2820\%2930752-2

Guba, E. G. \& Lincoln, Y. S. (1989).Fourth Generation Evaluation. Sage Publications.

Haynes, S. N, Richard, D. C. S. \& Kubany, E. S. (1995). Content validity in psychological assessment: a functional approach to concepts and methods. Psychol Assess, 7(3), 238-247. https://psycnet.apa.org/record/1996-03400-001. 
Jünger,S., Payne, S. A.: Brine, J., Radbruch, L. \&Brearley, S. G. (2017). Guidance on Conducting and REporting DElphi Studies (CREDES) in palliative care: Recommendations based on a methodological systematic review. Palliative Medicine, 31(8), 684-706. https://doi.org/10.1177/0269216317690685

Malta, D. C., Andrade, S. S. C. A., Oliveira, T. P., Moura, L., Prado, R. R., Souza, M. F. M. (2019). Probabilidade de morte prematura por doenças crônicas não transmissíveis, Brasil e regiões, projeções para 2025. Revista Brasileira de Epidemiologia. 22. https://doi.org/10.1590/1980-549720190030

Malta, D. C., França, E., Abreu, D. M. X., Perillo, R. D., Salmen, M. C., Teixeira, R, Passos, V., Souza, M. F. M., Mooney, M. \& Naghavi, M. (2017). Mortality due to non-communicable diseases in the Brazil, 1990 to 2015, according to estimates from the Global Burden of Disease study. São Paulo Med J 2017, 135(3): 213-21. https://doi.org/10.1590/1516-3180.2016.0330050117

Mendes, E. V. (2011). As redes de atenção à saúde. Organização Pan-Americana da Saúde. https://bvsms.saude.gov.br/bvs/publicacoes/r edes_de_atencao_saude.pdf

Menezes, M. C., Mingoti, S. A., Cardoso, C. S., Mendonça, R., \& Lopes, A. C. (2015). Intervention based on Transtheoretical Model promotes anthropometric and nutritional improvements - a randomized controlled trial. Eating behaviors, 17, 37-44. https://doi.org/10.1016/j.eatbeh.2014.12.007

Ministério da Saúde. (2010). Política Nacional de Promoção da Saúde. ( $3^{a}$ ed.). Secretaria de Vigilância em Saúde. Secretaria de Atenção à Saúde. https://bvsms.saude.gov.br/bvs/publicacoes/politica_nacional_promocao_saude_3ed.pdf

Ministério da Saúde. (2011). Plano de ações estratégias para o enfrentamento das doenças crônicas não transmissíveis (DCNT) no Brasil 2011-2022. Secretaria de Vigilância em Saúde, Departamento de Análise de Situação de Saúde. https://bvsms.saude.gov.br/bvs/publicacoes/plano_acoes_enfrent_dcnt_2011.pdf

Ministério da Saúde. (2012). Política Nacional de Atenção Básica. Secretaria de Atenção à Saúde, Departamento de Atenção Básica. http://189.28.128.100/dab/docs/publicacoes/geral/pnab.pdf

Ministério da Saúde. (2013). Política Nacional de Alimentação e Nutrição. (1 $1^{\mathrm{a}}$ ed.). Secretaria de Atenção à Saúde. Departamento de Atenção Básica. https://bvsms.saude.gov.br/bvs/publicacoes/politica_nacional_alimentacao_nutricao.pdf

Ministério da Saúde. (2014). Guia alimentar para a população brasileira (2a ed.). Secretaria de Atenção à Saúde, Departamento de Atenção Básica. https://bvsms.saude.gov.br/bvs/publicacoes/guia_alimentar_po pulacao_brasileira_2ed.pdf

Ministério da Saúde. (2016a). Instrutivo: Metodologias de Trabalho em Grupo para Promoção de Ações de Alimentação Adequada e Saudável na Atenção Básica. Ministério da Saúde, Universidade Federal de Minas Gerais.

https://bvsms.saude.gov.br/bvs/publicacoes/instrutivo_metodologia_trabalho_alimentacao_nutricao_atencao_basica.pdf

Ministério da Saúde. (2016b). Na cozinha com frutas, legumes e verduras. Ministério da Saúde, Universidade Federal de Minas Gerais. https://bvsms.saude.gov.br/bvs/publicacoes/cozinha_frutas_legumes_verduras.pdf

Ministério da Saúde. (2016c). Desmistificando Dúvidas sobre Alimentação e Nutrição - Material de Apoio para Profissionais de Saúde. Ministério da Saúde, Universidade Federal de Minas Gerais. https://bvsms.saude.gov.br/bvs/publicacoes/desmistificando_duvidas_sobre_alim enta\%C3 \% A7\%C3\%A3o_nutricao.pdf

Ministério do Desenvolvimento Social e Combate à Fome. (2012). Marco de referência de educação alimentar e nutricional para as políticas públicas. Secretaria Nacional de Segurança Alimentar e Nutricional. http:/www.mds.gov.br/webarquivos/arquivo/seguranca_alimen tar/caisan/Publicacao/E ducacao_Alimentar_Nutricional/1_marcoEAN.pdf

Organización Panamerica de la Salud (2019). Enfermedades no transmisibles: hechos y cifras. OPS. https://iris.paho.org/bitstream/handle/10665.2/51482/ OPSNMH19016_spa.pdf?sequence=2\&isAllowed=y

Pasquali, L. (2010) Instrumentação psicológica: Fundamentos e práticas. Artmed.

Pereira, R. D. M. \&Alvim, N. A. T. (2015).Delphi technique in dialogue with nurses on acupuncture as a proposed nursing intervention. Escola Anna Nery Revista de Enfermagem, 19(1), 174-80. https://doi.org/10.5935/1414-8145.20150024

Scarparo, A. F., Laus, A. M., Azevedo, A. L. C. S., Freitas, M. R. I., Gabriel, C. S. \& Chaves, L. D. P. (2012). Reflexões sobre o uso da técnica Delphi em pesquisas na enfermagem. Rev Rene, 13(1), 242-251. http://www.periodicos.ufc.br/rene/article/view/3803/3000

Silva, B. S., Coelho H. V., Cavalcante R. B., Oliveira V. C. \& Guimarães E. A. A. (2018). Estudo de avaliabilidade do Sistema de Informação do Programa Nacional de Imunização. Revista Brasileira de Enfermagem, 71(1), 660-669. https://doi.org/10.1590/0034-7167-2017-0601

Souza, E. F. D., Silva, A. G. \& Silva, I. L. F. (2018). Active methodologies for graduation in nursing: focus on the health care of older adults. Revista Brasileira de Enfermagem, 71(2), 976-980. https://doi.org/10.1590/0034-7167-2017-0150

Swinburn, B. A, Kraak, VI, Allender, S., Atkins, V. J., Baker, P. I., Bogard, J. R., Brinsden, H., Calvillo, A., Schutter, O., Devarajan, R., Ezzati, M., Friel, S., Goenka, S., Hammond, R. A., Hastings, G. , Hawkes, C., Herrero, M., Hovmand, P. S., Howden, M, \& Dietz, W. H. (2019), The Global Syndemic of Obesity, Undernutrition, and Climate Change: The Lancet Commission report. The Lancet, 393(10173), 791-846. https://doi.org/10.1016/S0140-6736(18)32822-8

Tanaka, O. Y. \& Tamaki, E. M. (2012). O papel da avaliação para a tomada de decisão na gestão de serviços de saúde. Ciência \& Saúde Coletiva, 17(4), 821828. http://dx.doi.org/10.1590/S1413-81232012000400002

Thurston, W \& Ramaliu. A. (2005).Evaluability assessment of a survivors of torture program: lessons learned. Canadian Journal of Program Evaluation, 20(2): 1-25. https://evaluationcanada.ca/secure/20-2-001.pdf

Timmins F. (2015).Surveys and questionnaires in nursing research. Nurse Standard, 29(42):42-50. https://doi.org/10.7748/ns.29.42.42.e8904

Trevisan, M. S. \& Huang, Y. M. (2003).Evaluability assessment: a primer. Practical Assessment, Research \& Evaluation, 8(20), 1-8. https://doi.org/10.7275/30ww-jr45 
Research, Society and Development, v. 10, n. 10, e600101019051, 2021

(CC BY 4.0) | ISSN 2525-3409 | DOI: http://dx.doi.org/10.33448/rsd-v10i10.19051

Wholey, J. S. (1977). Evaluability assessment. In: Rutman, L. (Ed.). Evaluation research methods: A base guide.(pp 41-56).

World Health Organization. (2020). World Health Statistics 2020. https://www.who.int/data/stories/leading-causes-of-death-and-disability-2000-2019-avisual-summary 2020 